\title{
Sheehan's syndrome in a dengue parturient
}

\author{
P Pooja ${ }^{1^{*},}$ N Dixit ${ }^{2}, K_{\text {Kilapadi }}^{3}$, A Sebastian $^{4}$, S Gopal $^{5}$ \\ Senior Resident ${ }^{1 *}$, Associate Professor ${ }^{2}$, Professor ${ }^{3}$, Postgraduate ${ }^{4}$, Undergraduate ${ }^{5}$ \\ St John's National Academy of Health Sciences, Karnataka, India.
}

\begin{abstract}
Dengue fever is a mosquito borne arboviral infection which is a major health problem worldwide. Complications vary widely and become critical mostly during defervescence period. Dengue fever in pregnant women can cause variety of obstetric and neonatal complications. Sheehan's syndrome (SS) is a parturitionrelated pituitary disease resulting from severe postpartum haemorrhage and can present with varying degrees of pituitary insufficiency. Emergency anaesthetic management of a dengue parturient and SS which developed subsequently, secondary to profuse intrapartum bleeding is discussed.
\end{abstract}

Keywords: Dengue fever; Sheehan's syndrome; HELLP syndrome

\section{Introduction}

Dengue fever is a common arboviral infection endemic to tropical and sub-tropical regions of the world. Its manifestations vary widely from a mild febrile illness to severe haemorrhagic shock with multiorgan failure. Risk of mortality from dengue fever is $2.5 \%^{1}$, higher if pregnant. Dengue in pregnancy can affect both mother and child, with complications including miscarriage, preeclampsia, increased risk of maternal haemorrhage, preterm delivery and low birth weight. ${ }^{2}$

Severe postpartum haemorrhage can lead to pituitary necrosis. Its clinical spectrum varies widely from simple fatigue to severe pituitary insufficiency leading to coma. ${ }^{3}$ Early diagnosis is the key to reduce morbidity and mortality associated with this condition. We report a case of severe dengue fever complicating labour in a 36wk pregnant mother leading to Sheehan's syndrome (SS) in the post-operative period.

*Correspondence: P Pooja

E mail: pooja.p22@gmail.com

iD https://orcid.org/0000-0003-3168-3142

Received: 05/11/2017

Accepted: 11/12/2017

DOI: http://doi.org/10.4038 /slja.v26i1.8286

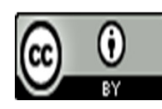

\section{Case report}

A 33y old lady at 36 weeks of gestation presented to emergency medicine department with history of fever for 7 days, headache and vomiting for 2 days. Vitals recorded were PR 110/min, RR 26/min, BP90/60mmHg and oxygen saturation $98 \%$ on room air. Non-stress test showed foetal distress and hence was taken for emergency LSCS. Initial investigations revealed haemoglobin of $16.9 \mathrm{~g} / \mathrm{dl}$, platelet count of $9000 \mathrm{~mm}^{3}$, and deranged liver enzymes in liver function test. Dengue serology was positive for NS1 Ag. Serum electrolytes, renal function tests and ECG were normal.

In view of low platelet count and foetal distress general anaesthesia with rapid sequence induction and endotracheal intubation was planned. Patient was premedicated with glycopyrrolate $0.2 \mathrm{mg}$ i.v., preoxygenated, induced with propofol $100 \mathrm{mg}$ and suxamethonium $100 \mathrm{mg}$ i.v. Cricoid pressure was maintained throughout and trachea intubated with $7.5 \mathrm{~mm}$ cuffed endotracheal tube. Anaesthesia was maintained with oxygen $(50 \%)$, air $(50 \%)$, intermittent doses of atracurium and isoflurane. After the delivery of the baby, fentanyl $1.5 \mu \mathrm{g} / \mathrm{kg}$ was administered i.v. and baby was shifted to NICU because of poor APGAR score. In view of severe thrombocytopaenia, 10 units of random donor platelets and 2 units of fresh frozen plasma were transfused. Intra-operatively she had two episodes of hypotension and oxygen saturation started to decline half hour post induction and ventilator parameters were adjusted accordingly. ABG showed $\mathrm{PaO}_{2}$ of $74 \mathrm{mmHg}$ and metabolic acidosis 
Pooja et al. Sri Lankan Journal of Anaesthesiology: 26(1):69-71(2018)

(7.24) despite $100 \% \mathrm{O}_{2}$ and PEEP of $10 \mathrm{mmHg}$. Intra-operatively blood loss was more than two litres for which two units of packed red blood cells were transfused. In view of severe blood loss, intraop hypoxaemia and metabolic acidosis, the patient was shifted to Intensive Care Unit (ICU) for further management.

In ICU, she was continued on mechanical ventilation. She continued to bleed during postoperative period for which Packed Red Blood Cells were transfused. Over next $48 \mathrm{hrs}$, her platelet count improved and bleeding was under control. Serial arterial blood gas analyses showed improved oxygenation. But she continued to be drowsy, despite been off sedation and was in altered sensorium which didn't improve till $5^{\text {th }}$ postoperative day. Hypoxic ischaemic encephalopathy was suspected; however, CT brain was normal. Pituitary hormone assay revealed low cortisol, FSH, LH, TSH and PRL. A diagnosis of SS was made based on peripartum history, clinical features and low hormone levels. Hormone replacement therapy was commenced with hydrocortisone $100 \mathrm{mg}$ and thyroxine $100 \mu \mathrm{g}$. By $7^{\text {th }}$ post-operative day, there was significant improvement in sensorium and she was extubated and shifted toward the next day.

\section{Discussion}

Dengue fever is caused by flavi group of viruses, aedes aegypti, a vector transmitting it to humans. Most of the states in India are dengue endemic. Symptomatic dengue infection can present with multisystem involvement causing severe degree of morbidity and mortality. The clinical spectrum is wide ranging from non-severe to severe manifestations. Incubation period is 2 weeks, with a sudden flare up of infection after this period. There are three phases: febrile, a febrile and recovery phase. Afebrile phase is the critical phase, where without timely intervention fatality rate is high. Once fever subsides there is significant plasma leak, haemorrhagic manifestations and multiorgan involvement, the warning signs being plasma leak, persistent vomiting and severe abdominal pain. Patients remain lethargic though mentally alert.

Our patient presented in the febrile phase with severe thrombocytopaenia, haemo concentration and clinical features suggestive of compensated shock. Anticipating massive intra operative bleeding, a pre-emptive transfusion of platelets was given. Early ARDS might be one of the possibilities for the desaturation observed intraoperatively. These events would have led to development of SS in the immediate postpartum period.

An ischaemic insult to the pituitary during parturition is postulated to be a factor in the pathogenesis of SS and its diagnosis is often delayed. Varying degree of pituitary dysfunction is known to occur and an early diagnosis is crucial in preventing morbidity and mortality. ${ }^{4}$ Diagnosis is based on laboratory studies, including hormone levels and hormone stimulation tests. Though our patient did not have typical features of SS, significant peripartum events and delayed recovery prompted us to investigate for SS. SS is one of the commonest causes of hypopituitarism in developing countries and often diagnosed late. Early recognition and prompt hormone replacement prevents morbidity. Late manifestations include characteristics of genital and axillary hair loss, reduced bone mineral density, signs of dry skin, pallor and other evidence of hyponatremia, diabetes insipidus and hypoglycaemia. ${ }^{5}$

Data on dengue in pregnant women is insufficient. Management is mainly supportive and revolves around fluid management, bleeding control and organ protective measures. ${ }^{6}$ One of the differential diagnoses for dengue in pregnancy is HELLP syndrome. There is overlapping of clinical features which include low platelet count and elevated liver enzymes. Unlike HELLP syndrome, dengue fever shows sero positivity and haemo concentration which were present in our patient. ${ }^{7}$

This case report outlines anaesthetic management of severe dengue complicating pregnancy and emergency operative delivery, to suspect SS in such patients. Though anaesthesia is known to complicate the disease process in such patients, this patient had uneventful recovery due to appropriate intensive care, early diagnosis and proper treatment with hormone replacement.

\section{References}

1. http://www.who.int/mediacentre/factsheets/fs117/e n/ accessed on 7th Feb 2017. 
2. Pouliot SH., Xiong X, Harville E et al. "Maternal dengue and pregnancy outcomes: a systematic review." Obstetrical \& gynecological survey 2010;65(2): 107-118.

PMid:20100360

3. Diri H, Karaca Z, Tanriverdi F, Unluhizarci K, Kelestimur F. Sheehan's syndrome: new insights into an old disease. Endocrine. 2016;51(1):22-31. https://doi.org/10.1007/s12020-015-0726-3 PMid:26323346

4. Kelestimur F. Sheehan's syndrome. Pituitary $2003 ; 6: 181-8$.

https://doi.org/10.1023/B:PITU.0000023425.20854 $.8 \mathrm{e}$

PMid:15237929

5. Kilicli F, Dokmetas HS, Acibucu F. Sheehan's syndrome, Gynecological Endocrinology 2013; 29(4), 292-295.

https://doi.org/10.3109/09513590.2012.752454

PMid:23245206

6. Chhabra A., Malhotra N. "Anesthetic management of a pregnant patient with dengue hemorrhagic fever for emergency cesarean section." International journal of obstetric anesthesia 2006;15(4): 306-310. https://doi.org/10.1016/j.ijoa.2006.04.011

PMid:16950613

7. Basurko C, Carles G, Youssef M, Guindi WEL. "Maternal and foetal consequences of dengue fever during pregnancy," European Journal of Obstetrics Gynecology\& Reproductive Biology 2009; 147(1): 29-32. https://doi.org/10.1016/j.ejogrb.2009.06.028

PMid:19632027 